\title{
Correction to: X-Ray CT
}

\section{Correction to:}

\section{H. Toda, $X$-Ray $C T$, https://doi.org/10.1007/978-981-16-0590-1}

The original version of the book was inadvertently published with the wrong copyright holder text in the book. This has now been updated and approved by the author. 